\title{
Unexpected Abundance of Pathological Tau in Progressive Supranuclear Palsy White Matter
}

\author{
Victoria Zhukareva, PhD, ${ }^{1}$ Sonali Joyce, BS, ${ }^{1}$ Teresa Schuck, BS, ${ }^{1}$ Vivianna Van Deerlin, MD, PhD, ${ }^{1}$ \\ Howard Hurtig, MD, ${ }^{2}$ Roger Albin, MD, ${ }^{3,4}$ Sid Gilman, MD, FRCP, ${ }^{3}$ Steven Chin, MD, ${ }^{5}$ Bruce Miller, MD, ${ }^{6}$ \\ John Q. Trojanowski, MD, PhD, ${ }^{1}$ and Virginia $\mathrm{M}-\mathrm{Y}$. Lee, $\mathrm{PhD}^{1}$
}

Objective: To investigate whether biochemical insoluble tau with 4 (4R) and/or 3 (3R) microtubule-binding repeats accumulate in white as well as gray matter in progressive supranuclear palsy (PSP), a neurodegenerative tauopathy.

Methods: To assess tau pathology in PSP white matter, we combined Western blot (WB) and immunohistochemical methods to analyze 23 autopsy-confirmed PSP brains.

Results: WBs showed an unexpected abundance of insoluble tau in white and gray matter of PSP brains, but biochemical tau pathology in white matter was not correlated with immunohistochemistry using the same panel of epitope-specific anti-tau antibodies used for WB. Despite heterogeneity in the representation of pathological 3R and 4R tau isoforms in cortical versus subcortical regions, biochemically detectable white matter tau pathology is a constant feature of PSP.

Interpretation: These studies show additional similarities between PSP and corticobasal degeneration, but unlike corticobasal degeneration, more abundant white matter tau pathology in PSP is detectable by WB than by immunohistochemistry. The differential detection of abnormal tau by biochemistry versus microscopy in PSP may reflect distinct pathological mechanisms, and elucidation of these processes will augment efforts to develop better strategies for the diagnosis and treatment of PSP and related neurodegenerative tauopathies.

Ann Neurol 2006;60:335-345

Progressive supranuclear palsy (PSP) is a neurodegenerative disorder characterized by postural instability, rigidity, parkinsonism, supranuclear gaze palsy, and cognitive abnormalities, including dementia, in association with prominent cortical and subcortical neuronal and glial tau pathology (reviewed in Cairns and colleagues, ${ }^{1}$ Dickson, ${ }^{2}$ Forman and colleagues, ${ }^{3}$ and McKhann and colleagues ${ }^{4}$ ). Thus, PSP shares phenotypic features with frontotemporal dementias and movement disorders that are classified as tauopathies. ${ }^{1-4}$ Tau phosphoproteins bind to microtubules, and their function is to promote the assembly and stability of microtubules, thereby playing a pivotal role in maintaining axonal transport (reviewed in Buee and colleagues, ${ }^{5}$ Lee and colleagues, ${ }^{6}$ and Roy and colleagues ${ }^{7}$ ). In the adult human central nervous system, six tau isoforms are produced by alternative splicing. Alternative splicing of exons 2 and 3 results in the insertion of $0(0 \mathrm{~N}), 1(1 \mathrm{~N})$, or $2(2 \mathrm{~N}) 29$ amino acid long motifs near the amino terminus, whereas splicing of exon 10 results in the inclusion of either 3 (3R) or 4 (4R) microtubule-binding domains in the carboxy terminus. From largest to smallest, the brain tau isoforms are designated $4 \mathrm{R} 2 \mathrm{~N}, 3 \mathrm{R} 2 \mathrm{~N}$, $4 \mathrm{R} 1 \mathrm{~N}, 3 \mathrm{R} 1 \mathrm{~N}, 4 \mathrm{R} 0 \mathrm{~N}$, and $3 \mathrm{R} 0 \mathrm{~N}$, respectively. The pathological filamentous tau inclusions in PSP are composed of aberrantly phosphorylated tau proteins, but there is a preferential accumulation of abnormal $4 \mathrm{R}$ tau isoforms in PSP. ${ }^{1-4}$ These tau inclusions are formed by neurons, astrocytes, and oligodendrocytes in PSP, but similar inclusions also occur in corticobasal degeneration (CBD), a 4R tauopathy that shares genetic and clinical features with PSP, although unlike PSP, there is abundant white matter tau pathol-
From the ${ }^{1}$ Department of Pathology and Laboratory Medicine, Center for Neurodegenerative Disease Research; ${ }^{2}$ Department of Neurology, University of Pennsylvania, Philadelphia, PA; ${ }^{3}$ Michigan Alzheimer's Disease Research Center, University of Michigan Health Center; ${ }^{4}$ Ann Arbor Affairs Medical Center Geriatric Research Education and Clinical Center, Ann Arbor, MI; ${ }^{5}$ Department of Pathology, University of Utah, Salt Lake City, UT; and ${ }^{6}$ Memory and Aging Center of the University of California, San Francisco, CA.

Received Jan 5, 2006, and in revised form Apr 25. Accepted for publication May 9, 2006.
This article includes supplementary materials available via the Internet at http://www.interscience.wiley.com/jpages/0364-5134/suppmat

Published online Jul 5, 2006, in Wiley InterScience (www.interscience.wiley.com). DOI: 10.1002/ana.20916

Address correspondence to Dr Lee, The Center for Neurodegenerative Disease Research, Department of Pathology and Laboratory Medicine, University of Pennsylvania School of Medicine, HUP/ Maloney 3, 3600 Spruce Street, Philadelphia, PA 19104-4283. E-mail: vmylee@mail.med.upenn.edu 


\begin{tabular}{|c|c|c|c|c|c|c|c|c|}
\hline $\begin{array}{l}\text { PSP } \\
\text { Cases }\end{array}$ & PMI (hr) & $\begin{array}{l}\text { Brain } \\
\text { Weight } \\
\text { (gm) }\end{array}$ & Sex & Onset & Duration & Dementia & $\begin{array}{l}\text { Family } \\
\text { History }^{\mathrm{a}}\end{array}$ & $\begin{array}{l}\text { 4R/3R Ratio } \\
\text { in Cortex }\end{array}$ \\
\hline 1 & 9.5 & 1,185 & M & 63 & 10 & ++ & No & $4 R>3 R$ \\
\hline 2 & 8.0 & 1,580 & M & 46 & 2 & + & Yes & $4 R>3 R$ \\
\hline 3 & 12.0 & 1,185 & $\mathrm{~F}$ & 79 & 2 & + & No & $4 \mathrm{R} \simeq 3 \mathrm{R}$ \\
\hline 4 & 19.0 & 1,240 & $\mathrm{~F}$ & 68 & 7 & + & No & $4 R>3 R$ \\
\hline 5 & 6.5 & 1,247 & $\mathrm{~F}$ & 65 & 7 & + & No & $4 \mathrm{R} \simeq 3 \mathrm{R}$ \\
\hline 6 & 10.5 & 1,380 & M & 69 & 4 & + & No & $4 \mathrm{R} \simeq 3 \mathrm{R}$ \\
\hline 7 & 4.5 & 1,190 & $\mathrm{~F}$ & 62 & 7 & ++ & No & $4 R \simeq 3 R$ \\
\hline 8 & 19.0 & 1,324 & $\mathrm{~F}$ & 67 & 11 & + & No & $4 \mathrm{R} \simeq 3 \mathrm{R}$ \\
\hline 9 & 19.5 & 1,308 & M & 68 & 6 & + & No & $4 \mathrm{R} \simeq 3 \mathrm{R}$ \\
\hline 10 & 10.0 & 1,136 & $\mathrm{~F}$ & 80 & 2 & + & No & $4 R \simeq 3 R$ \\
\hline 11 & 5.0 & 1,340 & M & 71 & 10 & + & No & $4 \mathrm{R} \simeq 3 \mathrm{R}$ \\
\hline 12 & 5.5 & 1,160 & $\mathrm{~F}$ & 71 & 8 & + & Yes & $4 R>3 R$ \\
\hline 13 & 6.0 & 1,170 & $\mathrm{~F}$ & 68 & 7 & ++ & No & $4 R \simeq 3 R$ \\
\hline 14 & 10.0 & 1,450 & M & 74 & 7 & + & No & $4 \mathrm{R} \simeq 3 \mathrm{R}$ \\
\hline 15 & NA & 1,276 & $\mathrm{~F}$ & & & + & No & $4 R \simeq 3 R$ \\
\hline 16 & 4.0 & 1,430 & M & 75 & 9 & + & No & $4 R \simeq 3 R$ \\
\hline 17 & 6.0 & 1,060 & M & 81 & 6 & ++ & No & $4 R \simeq 3 R$ \\
\hline 18 & 16.5 & 1,064 & $\mathrm{~F}$ & 79 & 11 & ++ & No & $4 R>3 R$ \\
\hline 19 & 4.0 & 1,234 & $\mathrm{~F}$ & 66 & 6 & ++ & Yes & $4 R \simeq 3 R$ \\
\hline 20 & 2.0 & 1,350 & M & 81 & 15 & ++ & No & $4 \mathrm{R} \simeq 3 \mathrm{R}$ \\
\hline 21 & NA & 1,240 & M & 63 & 4 & ++ & Yes & $4 R>3 R$ \\
\hline 22 & 2.0 & NA & $\mathrm{F}$ & 71 & 6 & ++ & Yes & $4 \mathrm{R}>3 \mathrm{R}$ \\
\hline 23 & 7.0 & 1,332 & $\mathrm{~F}$ & 68 & 4 & + & No & $4 R>3 R$ \\
\hline CBD 1 & 6.5 & 1,141 & $\mathrm{~F}$ & 64 & 7 & + & No & $4 R>3 R$ \\
\hline CBD 2 & 5.0 & 1,182 & $\mathrm{~F}$ & 62 & 8 & + & No & $4 \mathrm{R} \geq 3 \mathrm{R}$ \\
\hline $\mathrm{AD} 1$ & 4.5 & 1,019 & $\mathrm{~F}$ & 79 & 8 & ++++ & No & $4 R \simeq 3 R$ \\
\hline $\mathrm{AD} 2$ & 8.0 & 1,062 & M & 80 & 6 & ++++ & No & $4 R \simeq 3 R$ \\
\hline $\mathrm{AD} 3$ & 5.0 & 1,100 & M & 79 & 5 & ++++ & No & $4 R \simeq 3 R$ \\
\hline
\end{tabular}

Twenty-three progressive supranuclear palsy (PSP), two corticobasal degeneration (CBD), and three Alzheimer's disease (AD) patients were used for this study. In 21 of 23 cases, tau haplotypes were identified as $\mathrm{H} 1 / \mathrm{H} 1$ and A0/A0 (data not shown).

${ }^{a}$ Family members other than a proband were diagnosed with neurodegenerative disorder.

${ }^{\mathrm{b}} 4 \mathrm{R}$ and $3 \mathrm{R}=$ four and three microtubule-binding domains in the tau molecule correspondingly. The ratio $4 \mathrm{R} / 3 \mathrm{R}$ was assessed based on semiquantitative evaluation of Western blot analyses.

PMI $=$ postmortem interval, hours; $\mathrm{NA}=$ information is not available; $+=$ mild cognitive impairment; $++=$ progressive dementia, $++++=$ severe dementia.

ogy in $\mathrm{CBD}$ that can be detected by tau immunohistochemistry (IHC). ${ }^{1-4,8-11}$

However, because the presence of pathologically insoluble tau detected with biochemical methods in a neurodegenerative tauopathy may not be evident microscopically as tau inclusions, ${ }^{12,13}$ we examined this possibility in PSP. To do this, we analyzed tau pathology in 23 clinically and neuropathologically confirmed PSP cases with respect to the distribution of tau lesions and the biochemical profile of abnormal tau isoforms in multiple cortical gray and white matter regions, as well as subcortical brain regions. Remarkably, these studies demonstrated an unexpected abundance of tau pathology in white matter that was detected biochemically, but not by microscopic examination using the same panel of epitope-specific tau antibodies. Although this reflects incompletely understood differences in the pathological modifications of tau in PSP, we anticipate that clarification of this conundrum will accelerate efforts to improve the diagnosis and treatment of PSP and related tauopathies.

\section{Materials and Methods}

\section{Brain Tissue Samples}

Brain tissue samples used in this study were obtained from the Alzheimer's Disease Centers of the University of Pennsylvania School of Medicine, the Columbia University College of Physicians and Surgeons, the University of Michigan, and the University of California San Francisco. The diagnostic clinical and neuropathological assessment of all cases was performed according to accepted guidelines, and patients with PSP, Alzheimer's disease (AD), and CBD met consensus criteria for clinical and neuropathological diagnosis as described previously. ${ }^{4,9,14-18}$ Demographic and other key clinical information on the subjects studied here are summarized in the Table. 


\section{Frontal Ctx. \\ gray matter PSP-17}
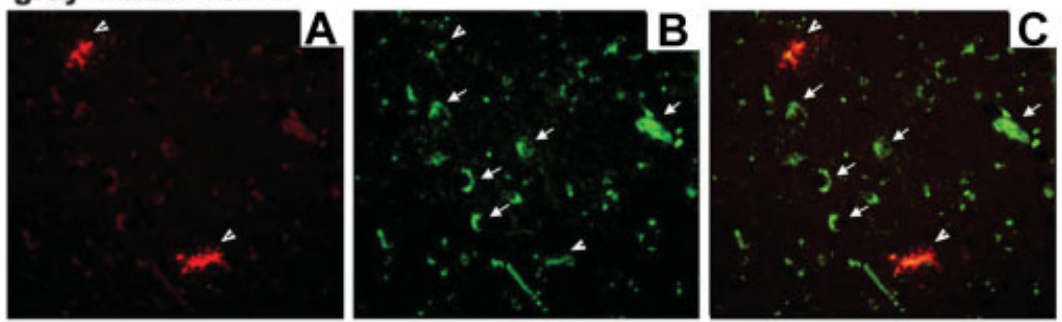

\section{gray matter PSP-19}
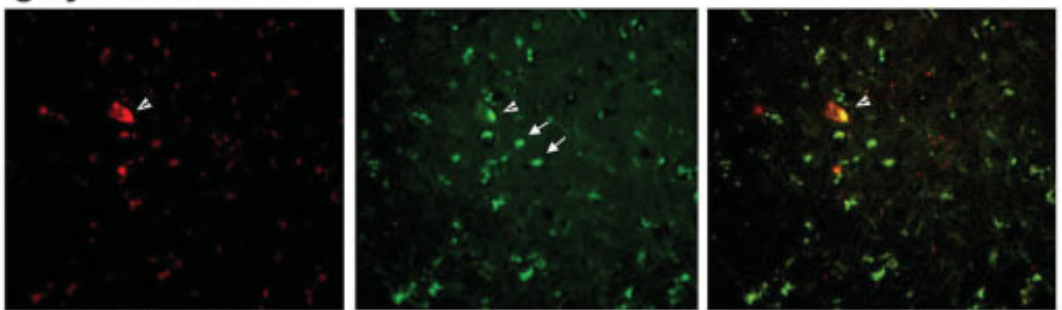

white matter PSP-19
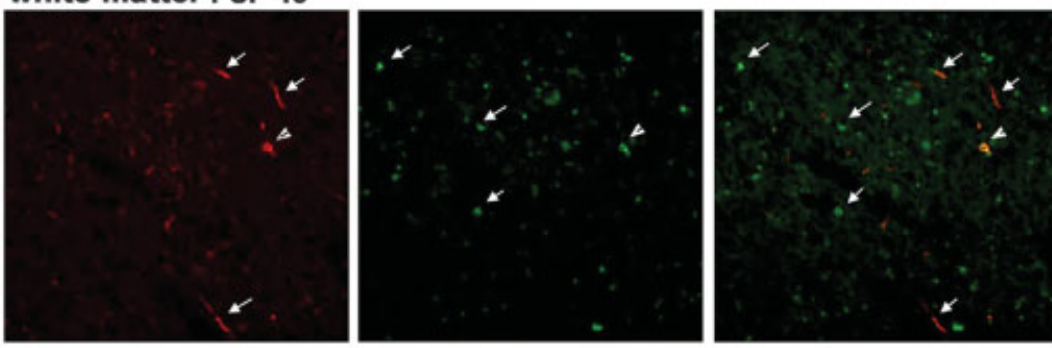

Medulla PSP-17
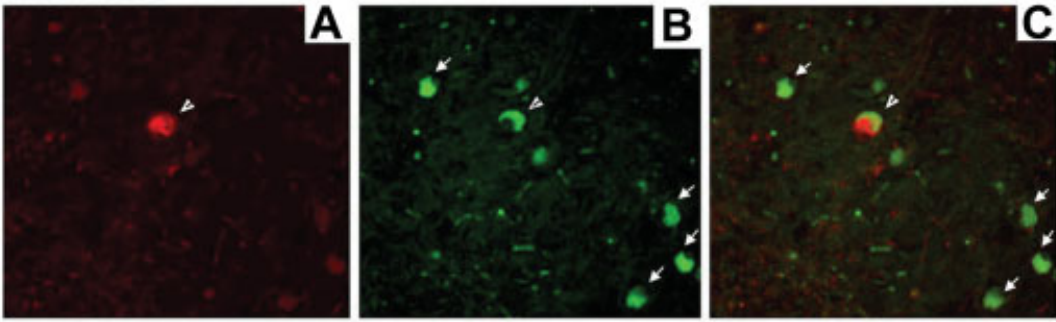

PSP-19
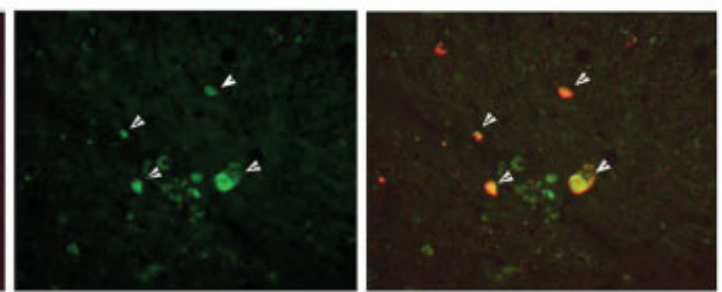

Fig 1. Comparative immunohistochemical (IHC) analysis of tau pathology in progressive supranuclear palsy (PSP). Single- and double-label immunoflourescence staining to show the individual localization $(A, B)$ and coexpression $(C$, overlay) of phosphorylation-dependent $\left(P_{d}\right)$ PHF1 and phosphorylation-independent $\left(P_{i}\right) N$-tau epitopes in frontal cortex and medulla of PSP brains. Numerous PHF1 (red) and N-tau-positive (green) lesions were detected in the frontal cortex and medulla from two PSP cases (1 and 2). Although individual immunolabeling with PHF1 or N-tau showed numerous tau inclusions throughout the cortical gray matter (arrows; $A, B$ ), both tau epitopes were colocalized only to a small subset of cells (arrowheads; $C$, overlay). In comparison with $N$-tau antibodies, PHF1 labeled less tau inclusions. In the cortical white matter, PHF1 and N-tau immunoreactivity was confined to a subset of single-labeled threadlike structures and to sparsely distributed glial cells. In medulla, colocalization of PHF1 and $N$-tau epitopes varied from partial overlap in a subset of neurons (Case 1) to numerous double-labeled cells (Case 2). 


\section{Immunohistochemistry}

Tissue samples were collected at autopsy and fixed in 10\% neutral-buffered formalin. The brain regions examined here included frontal, temporal, parietal, and occipital lobes; cerebellum; basal ganglia; and the brainstem after samples were paraffin embedded and processed for neuropathological assessment. Also, frozen tissue from areas adjacent to blocks of frozen brain used for biochemical analyses (see later) were similarly fixed in neutral-buffered formalin, paraffin embedded, and examined as described here and in previous studies. ${ }^{9,12,14,16-18}$ To do this, we cut $6 \mu \mathrm{m}$-thick sections and assessed them with and without antigen retrieval methods (eg, with formic acid $[\mathrm{FA}]$ pretreatment) for the presence of tau pathology using a panel of previously described epitopespecific and other tau antibodies, ${ }^{9,12,14,16-19}$ including the phosphorylation-independent $\left(\mathrm{P}_{\mathrm{i}}\right)$ rabbit polyclonal antirecombinant human tau antibody (17025; dilution $1: 10,000)$ and rabbit polyclonal antibody raised against the peptide [AEPRQEFEVMEC] at the $\mathrm{N}$ terminus of human tau (N-tau; 1:1,000). In addition, a phosphorylationdependent $\left(\mathrm{P}_{\mathrm{d}}\right)$ tau monoclonal antibody (MAb) PHF1 (1: 500; a gift from Dr P. Davies) also was used for IHC analysis, whereas an affinity-purified rabbit polyclonal exon 10specific (E10; dilution 1:1,000) tau antibody was used to demonstrate the distribution of $4 \mathrm{R}$ tau isoforms in tau lesions in PSP. Double-immunofluorescence IHC was conducted to determine the localization of $\mathrm{N}$-tau and PHF1 epitopes in tau lesions by previously described methods ${ }^{19}$ using donkey anti-rabbit fluorescein isothiocyanate-conjugated or donkey anti-mouse TxR-conjugated secondary antibodies (Jackson ImmunoResearch Laboratories, West Grove, PA) diluted 1:100 and 1:200 in phosphate-buffered saline, respectively. Finally, Gallyas silver staining was performed to detect fibrillary lesions, as described previously., ${ }^{9,12,14,16-19}$

\section{Biochemical and Quantitative Western Blot Analyses}

Frozen tissue samples from multiple cortical and subcortical brain regions of most cases were used for the biochemical analysis. Gray and white matter regions were dissected and processed separately as described previously. ${ }^{9,12-14,18,19}$ Soluble and sarkosyl-insoluble tau proteins were prepared and dephosphorylated using alkaline phosphatase from Escherichia coli (Sigma, St. Louis, MO), as described previously. ${ }^{9}, 12-14,18,19$ Samples were resolved on $7.5 \%$ sodium dodecyl sulfate (SDS) polyacrylamide gel electrophoresis (PAGE). Nitrocellulose replicas from each gel were probed with a mixture of $\mathrm{P}_{\mathrm{i}}$ anti-tau MAbs T14 (dilution 1:3,000; $\mathrm{N}$-terminal, 141-178 residues) and T46 (dilution 1:1,000; C-terminal, 404-441 residues). For phosphoepitope analysis of insoluble tau in nondephosphorylated samples, the following MAbs were used: phosphorylation-dependent $\left(\mathrm{P}_{\mathrm{d}}\right)$ antibodies PHF1 (dilution 1:1,000; Ser396/Ser404) and AT8 (dilution 1:1,000; Ser202/Thr181 Innogenetics, Belgium). Bound antibodies were visualized using Enhanced Chemiluminescence (ECL) kits (Perkin-Elmer, Oak Brook, IL) according to the manufacturer, and after stripping, the same nitrocellulose replicas were reprobed with other anti-tau antibodies. For quantitative Western blot analysis, anti-mouse $\left[\mathrm{I}^{1}{ }^{125}\right]-\mathrm{IgG}$ was used, and quantitative data were generated using ImageQuant analysis software
(Molecular Dynamics, G.E. Healthcare, USA) as described previously. ${ }^{9,12-14,18,19}$ For regional analysis of tau pathology, final data were presented as a ratio of a relative amount of insoluble tau isolated from each brain region compared with that in the basal ganglia, wherein are abundant amounts of sarkosyl-insoluble tau in PSP brains Antibodies, 17025, N-Tau, E10, T14 and T46 were generated from our laboratory.

\section{Sequential Extraction of Tau Proteins}

Selected brain areas were used for the sequential extraction of tau proteins with buffers/solutions of increasing stringency, as described previously. ${ }^{12,13,18,19}$ The following buffers with a mixture of protease inhibitors were used: (1) high-salt buffer $(0.75 \mathrm{M} \mathrm{NaCl}, 50 \mathrm{mM}$ tris(hydroxymethyl)aminomethane [Tris] buffer, 2mM EDTA, pH 7.4; (2) 1\% Triton X-100 in Buffer 1 (Sigma); (3) radioimmunoprecipitation assay buffer $(0.1 \%$ SDS, $1 \%$ NP- $40,0.5 \%$ sodium deoxycholate, $2 \mathrm{mM}$ EDTA, $150 \mathrm{mM} \mathrm{NaCl}, 50 \mathrm{mM}$ Tris buffer, $\mathrm{pH}$ 8.0); (4) 2\% SDS; and (5) 80\% FA. Each extraction step was repeated twice. After each extraction, samples were spun at $45,000 \mathrm{~g}$ for 30 minutes at $4^{\circ} \mathrm{C}$, after which supernatants were collected and subjected to Western blot analysis. Protein concentrations were adjusted between samples from each extraction.

\section{Apolipoprotein Genotyping and Tau Haplotype Analyses}

DNA was isolated from brain tissue using a commercial kit (Qiagen, Valencia, CA). Apolipoprotein E genotyping was performed by using a previously described polymerase chain reaction restriction fragment length polymorphism method. ${ }^{14,20}$ Tau gene (microtubule-associated protein tau [MAPT]) haplotype analysis was performed by polymerase chain reaction amplification of exon 9, followed by restriction digestion with HpaII and HpyCH4IV to genotype for the three known polymorphisms of exon 9 that segregate with the known major MAPT haplotypes. ${ }^{21,22}$

\section{Results}

\section{Immunohistochemical Distribution of Tau Pathology in Progressive Supranuclear Palsy Brains}

The distribution of tau lesions in cortical and subcortical regions of PSP brains has been well described, and the IHC observations here concurred with data from previous studies as illustrated in four representative PSP cases showing morphologically evident tau lesions that were more abundant in gray versus white matter regions (data not shown), but brainstem and basal ganglia were most affected. This distribution was confirmed in Gallyas-stained preparations, and these findings contrasted with comparable IHC data from CBD brains wherein cortical, as well as subcortical, white matter showed abundant tau pathology by light microscopy (data not shown). Double-immunofluorescence IHC using $\mathrm{P}_{\mathrm{i}} \mathrm{N}$-tau (green) and $\mathrm{P}_{\mathrm{d}}$ PHF1 (red) antibodies (Fig 1) showed that neuronal and glial tau inclusions in PSP were immunopositive for both antibodies, al- 


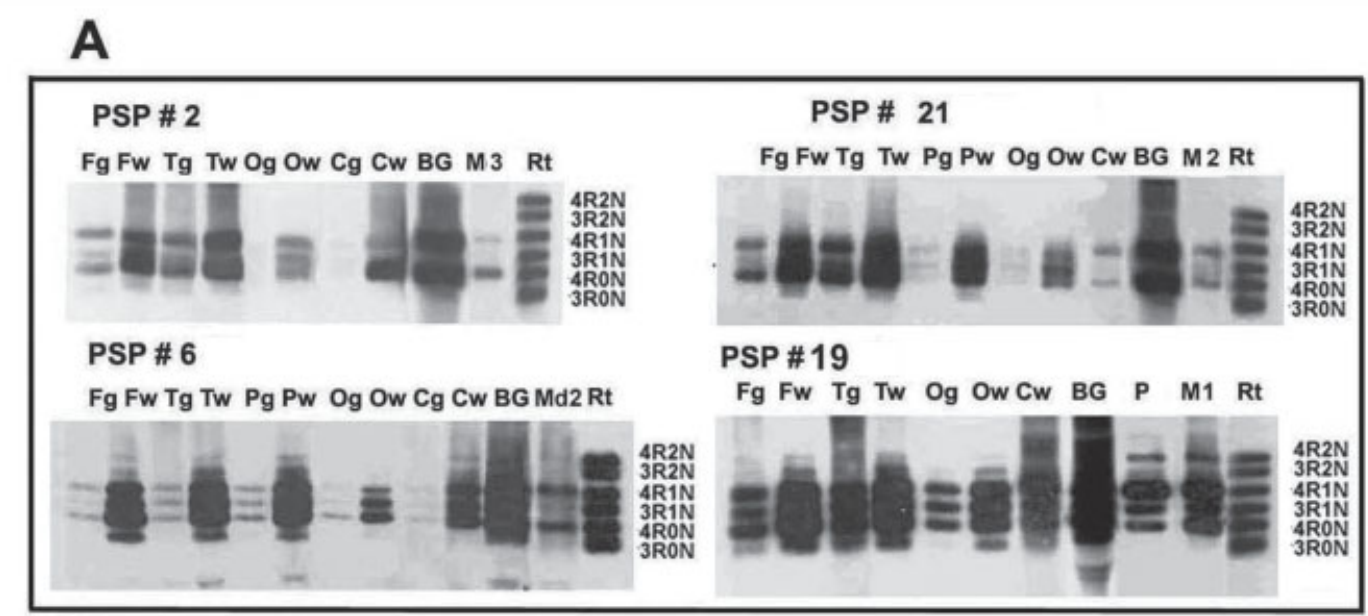

B

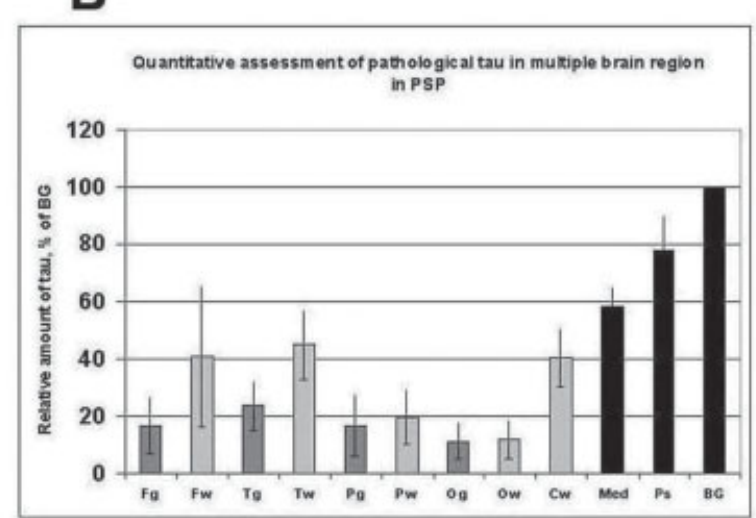

C
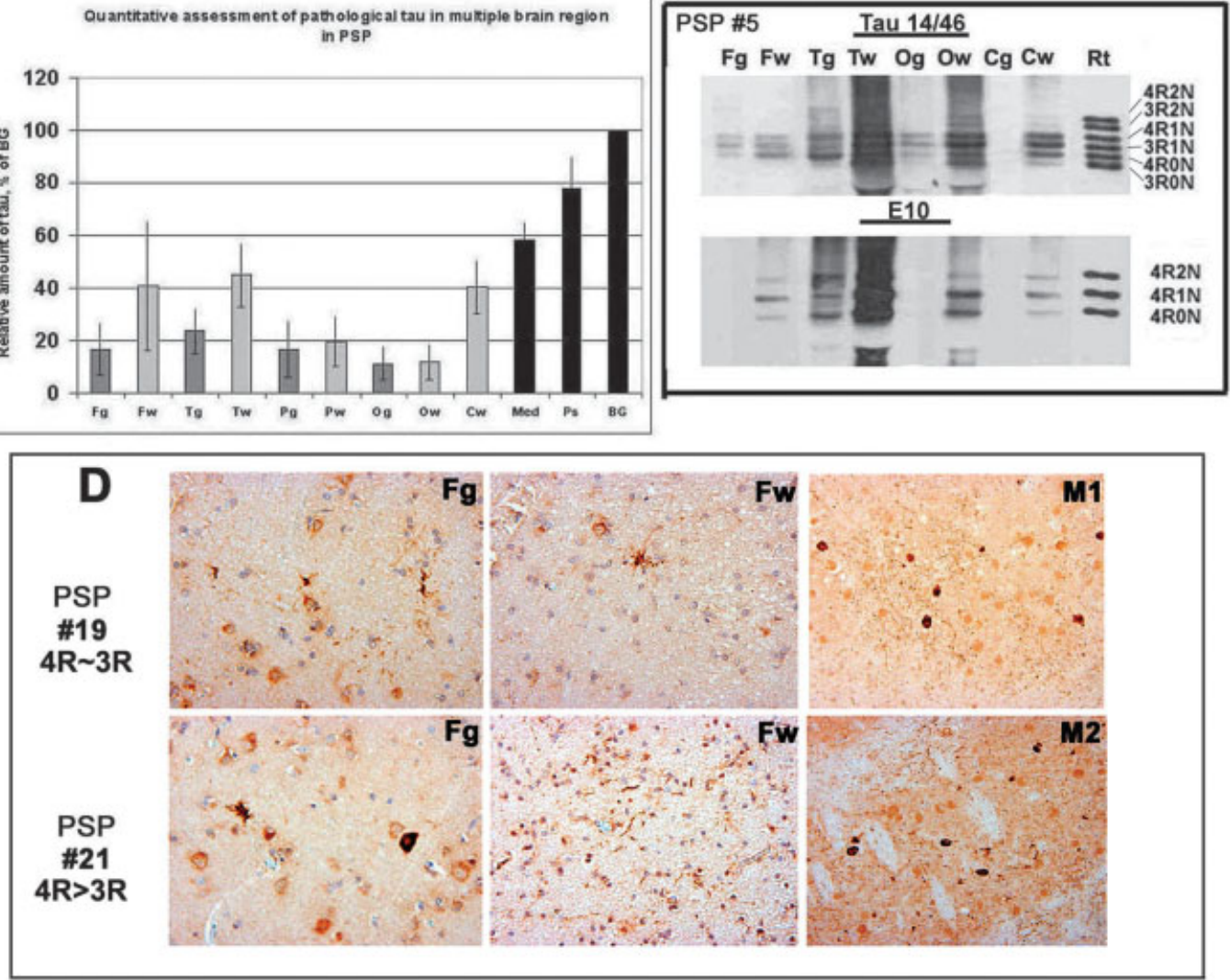

Fig 2. Quantitative Western blot analysis of pathological tau proteins in progressive supranuclear palsy (PSP). Aliquots of sarkosyl-insoluble fractions from multiple brain regions were dephosphorylated and resolved on 7.5\% SDS-PAGE. Mixture of six recombinant human tau proteins (Rt) were used as standards. To estimate total insoluble tau pool, we probed nitrocellulose replicas with a mixture of monoclonal antibodies (MAbs) T14 and T46. (A). Brain region-specific distribution of pathological tau in four PSP cases. The amounts of insoluble tau were variable among cases, but they were consistently higher in cortical white matter of PSP brains. (B). Quantitative assessment of sarkosyl-insoluble tau in different brain regions. For quantitative Western blot analysis, anti-mouse $\left[I_{-}^{125}\right]$-IgG was used, and quantitative data were generated using ImageQuant analysis. Each bar represents a relative amount of insoluble tau isolated from $1 \mathrm{gm}$ of PSP brain tissue as a percentage of a total amount of the latter in PSP basal ganglia. (C). The nitrocellulose replica here was subjected to Western blot analysis using a mixture of MAbs, Tau14 and Tau46 (top panels), after which the same membrane was stripped and reprobed with rabbit polyclonal E10-specific tau antibody that selectively recognizes all $4 R$ tau isoforms in the PSP samples and in Rt mixture (bottom panels). (D). Immunohistochemical (IHC) analysis of two representative PSP cases with similar levels of pathological $4 R$ and $3 R(4 R \sim 3 R$ ) isoforms or predominantly $4 R$ tau $(4 R>3 R)$ isoforms. $B G=$ basal ganglia; $C t x=$ cortex; $F g=$ frontal cortex, gray matter; Fw $=$ white matter; M1, 2, 3 = medulla levels 1, 2, and 3 dissected for study as per the diagram in Figure 3; Og = occipital cortex, gray matter; $O w=$ occipital cortex, white matter; $P=$ pons; $P g=$ parietal cortex, gray matter; $P w=$ parietal cortex, white matter; Tg $=$ temporal cortex, gray matter; $T w=$ temporal cortex, white matter. 
though PHF1 and N-tau immunoreactivity only colocalize about 25 to $50 \%$ of the time, and the $\mathrm{N}$-tau antibody generally detected more tau lesions than PHF1.

\section{Biochemical Assessment of Pathological Tau in Progressive Supranuclear Palsy}

Western blot analysis of sarkosyl-insoluble tau fractions from multiple cortical and subcortical regions was performed to evaluate the extent of tau pathology in PSP brains and to compare these data with the IHC findings from the same brain regions. All samples were dephosphorylated using alkaline phosphatase from E. coli, and representative images of nitrocellulose replicas probed with a mixture of $\mathrm{P}_{\mathrm{i}}$ MAbs T46/T14 are shown in Figure 2A. In all PSP cases, sarkosyl-insoluble tau was most abundant in basal ganglia and brainstem compared with cortical regions; this is consistent with IHC studies reported here and elsewhere. ${ }^{2,5,8,13}$ A similar biochemical tau profile was also detected by 17025 and $\mathrm{N}$-tau rabbit polyclonal antibodies (data not shown).

A quantitative Western blot tau profile for PSP was obtained, and the relative amounts of extracted insoluble tau from each brain region were presented as a percentage of the latter in basal ganglia (see Fig 2B). Unexpectedly, in more than $70 \%$ of PSP cases (19/23), the amount of insoluble tau in the cortical white matter was variable, but appreciably higher than in the gray matter from the same brain regions despite a low abundance of IHC-detectable tau inclusions in sections adjacent to the regions examined by Western blots here. Thus, although our IHC data confirm the previously reported abundance of microscopic tau pathology in PSP gray matter and the paucity of tau lesions in PSP white matter, our novel biochemical data demonstrate a previously unrecognized accumulation of pathological tau in PSP white matter.

Dephosphorylated samples from different brain regions were used to characterize the representation of $3 \mathrm{R}$ and $4 \mathrm{R}$ tau isoforms in soluble and sarkosylinsoluble fractions from different PSP cases. Analysis of tau isoforms in multiple brain regions from 23 PSP cases showed a variable mixture of $3 \mathrm{R}$ and $4 \mathrm{R}$ tau isoforms in the cortical regions in 15 cases, whereas $4 \mathrm{R}$ tau species were overrepresented in 7 cases (see Fig 2A). Rabbit polyclonal E10-specific tau antibody demonstrated $4 \mathrm{R}$ tau isoforms in sarkosyl-insoluble preparation and the extent of pathology in E10-positive tau lesions (see Fig 2C). Furthermore, although all six tau isoforms were detected by a mixture of T14 and T46 MAbs (see Fig 2C), E10-specific antibody selectively recognized only $4 \mathrm{R}$ tau isoforms in each sample and in the standard mixture of human recombinant tau proteins. IHC analysis of two PSP cases with $4 \mathrm{R}$ approximately the same as $3 \mathrm{R}$ and with $4 \mathrm{R}$ greater than $3 \mathrm{R}$ tau isoforms composition is shown in Figure 2D, and in both of these cases, E10-immunopositive neurofibrillary tangles in neurons, as well as glial tau inclusions and threads, were detected in cortical gray and white matter, as well as in brainstem. In PSP cases with $4 \mathrm{R}$ isoform overexpression, the number of E10-positive structures, particularly in the white matter, was notably higher.

Predominant expression of $4 \mathrm{R}$ tau isoforms was a distinct signature of pathological tau in brainstem structures, and in a subset of PSP cases, the $4 \mathrm{R} 2 \mathrm{~N}$ tau pathological isoform was more prominent than other tauopathies (Figs 3A, B). Interestingly, in the brainstem from $\mathrm{AD}$ brains, insoluble tau profile recapitulated a cortical pattern of tau isoforms with all six isoforms being present (see Fig 3A, bottom panel). To determine whether the ratio of tau isoforms is altered for normal tau in PSP, we evaluated soluble fractions from the cortex and the brainstem in PSP cases using a quantitative Western blot analysis. In contrast with differential overrepresentation of $4 \mathrm{R}$ tau isoforms in sarkosyl-insoluble fractions from the cortex and the brainstem, the 4R:3R tau isoform ratio in corresponding soluble fractions from different PSP cases was approximately 1 (see Figs $3 \mathrm{Aa}$ and $3 \mathrm{Ab}$ ). Finally, we compared the biochemical profile of spinal cord tau pathology in PSP with that in AD, which showed that the amounts of pathological tau proteins in PSP spinal cord were higher than in $\mathrm{AD}$ spinal cord, and there was a predominance of $4 \mathrm{R}$ tau in PSP spinal cord, whereas $\mathrm{AD}$ spinal cord showed similar amounts of $3 \mathrm{R}$ and $4 \mathrm{R}$ tau (see Fig 3C). Similar biochemical studies were performed on cortical gray and white matter from prospectively followed cognitively normal individuals, and these studies confirmed the absence of pathological tau proteins in these normal brain samples (see Supplementary Figure).

Representative Western blots comparing sarkosylinsoluble tau extracted from PSP, CBD, and AD brains are shown in Figure 4, where distinct profiles of pathological tau for each disorder are evident. For example, in gray matter of $\mathrm{AD}$ cortex, the amounts of pathological tau were substantially higher than in PSP and $\mathrm{CBD}$ cortices, whereas in adjacent $\mathrm{AD}$ white matter, it was more moderate. Furthermore, cortical tau pathology in PSP was more moderate than $\mathrm{AD}$ or $\mathrm{CBD}$, and the amount of pathological tau in PSP white matter was substantially higher than in the corresponding gray matter, which contrasts sharply with our IHC data on microscopically evident tau lesions (see Figs 1A-C). In CBD, the amounts of sarkosylinsoluble tau correlated well with abundant taupositive inclusions in both anatomical regions (see Figs 1 and $4 \mathrm{~B}$ ). Furthermore, representative Western blots of tau in fractions sequentially extracted with buffer of increasing strength (ie, from high-salt buffer to FA) 
A

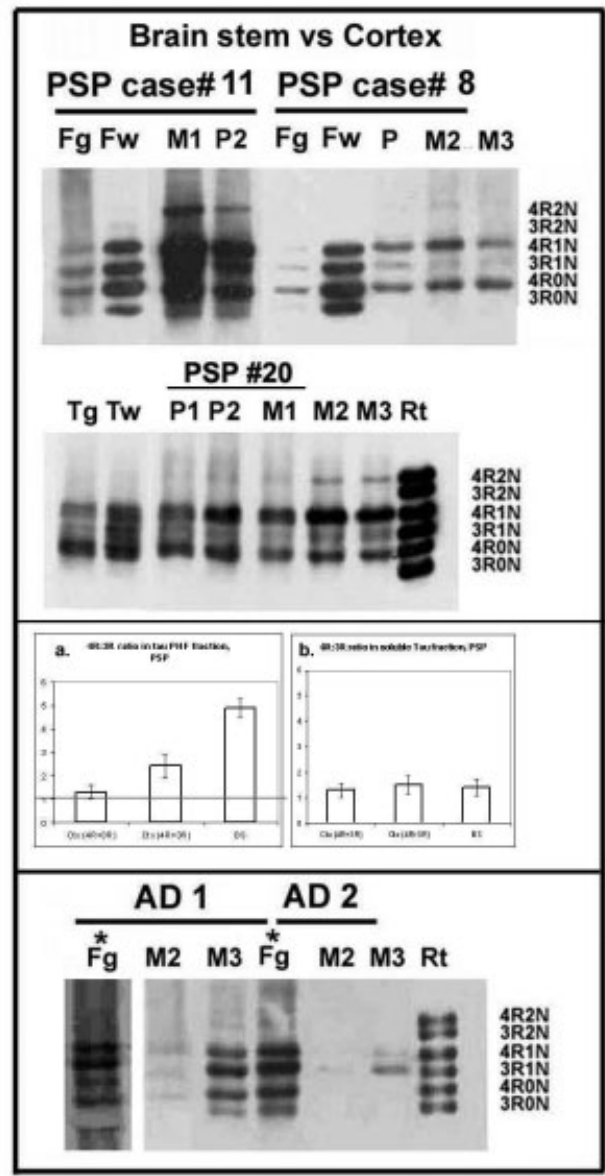

B

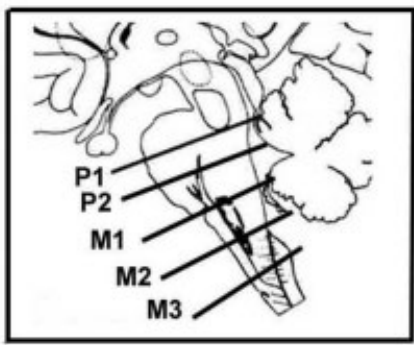

C

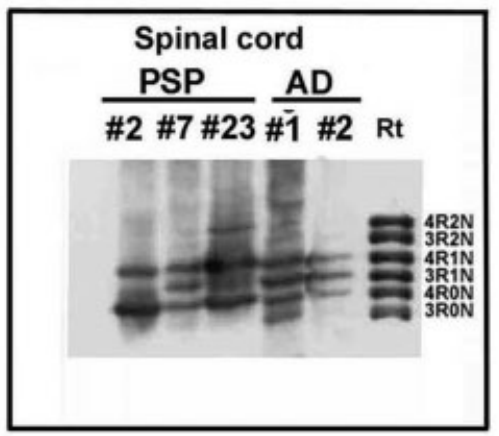

Fig 3. The biochemical profile of pathological tau proteins differs in subcortical versus cortical regions of the progressive supranuclear palsy (PSP) brain. Sarkosyl-insoluble tau was extracted from different regions of the PSP brainstem and spinal cord, as well as from Alzheimer's disease (AD) cases. (A, top panel) Western blot analysis of tau proteins in PSP brainstem and cortex. The total amounts of insoluble tau in the PSP brainstem are higher than in PSP cortex, and $4 R$ tau isoforms (4R2N, 4R1N, 4RON) predominate. (Middle panel) Quantitative Western blot analysis of soluble and insoluble tau in PSP. (Bottom panel) Comparative analysis of pathological tau in the brainstem and frontal cortex of two $A D$ patients ( $A D 1$ and $A D 2$ ). Asterisks identify lanes in which the amount of sample from $A D$ cortex loaded onto the gel was only $20 \%$ of that from PSP cortex. (B) Schematic diagram of the brainstem to show the different levels at which samples were taken for study from the pons (P1 and P2) and medulla (M1-3). (C) Biochemical profile of spinal cord tau pathology in PSP $(n=3)$ and $A D(n=2)$. The amounts of pathological tau proteins in PSP spinal cord were higher than in $A D$ spinal cord, and there is a predominance of $4 R$ tau in PSP spinal cord, whereas $A D$ spinal cord shows similar amounts of $3 R$ and $4 R$ tau. $F g=$ frontal cortex, gray matter; $F w=$ frontal cortex white matter; $M 1,2,3=$ medulla levels 1,2, and $3 ; P=$ pons; $T g=$ temporal cortex, gray matter; $T w=$ temporal cortex, white matter.

from the temporal cortex of PSP, CBD, and AD case are shown in Figure 5. These data show no significant difference in the soluble tau fractions among PSP, $\mathrm{CBD}$, and $\mathrm{AD}$, but there is a distinctive tau solubility profile in radioimmunoprecipitation assay, SDS, and FA fractions from each of these three tauopathies. Thus, the significant differences in quantities of insoluble tau detected in PSP, CBD, and AD suggest there is a differential solubility of pathological tau species in these tauopathies.

To further characterize the biochemical properties of pathological tau proteins in cortical gray and white matter in PSP, we analyzed the distribution of tau phosphoepitopes in different brain regions in PSP (Fig 6). Nondephosphorylated sarkosyl-insoluble samples were used for Western blot analysis. A mixture of tau-specific $\mathrm{P}_{\mathrm{i}}$ MAbs, T14/T46 was used to estimate a total sarkosyl-insoluble tau pool in the gray and white matter in two PSP cases. To demonstrate the relative level of site-specific phosphorylation and to compare it with the total amounts of insoluble tau, we reprobed the same nitrocellulose replicas with $P_{d}$ MAbs. PHF1 specific for Ser-396/404 and AT8 that recognize Ser202/Thr-205 detected predominantly 64 and $68 \mathrm{kD}$ tau species. Also, although the amounts of T14/T46-positive sarkosyl-insoluble tau in the corti- 


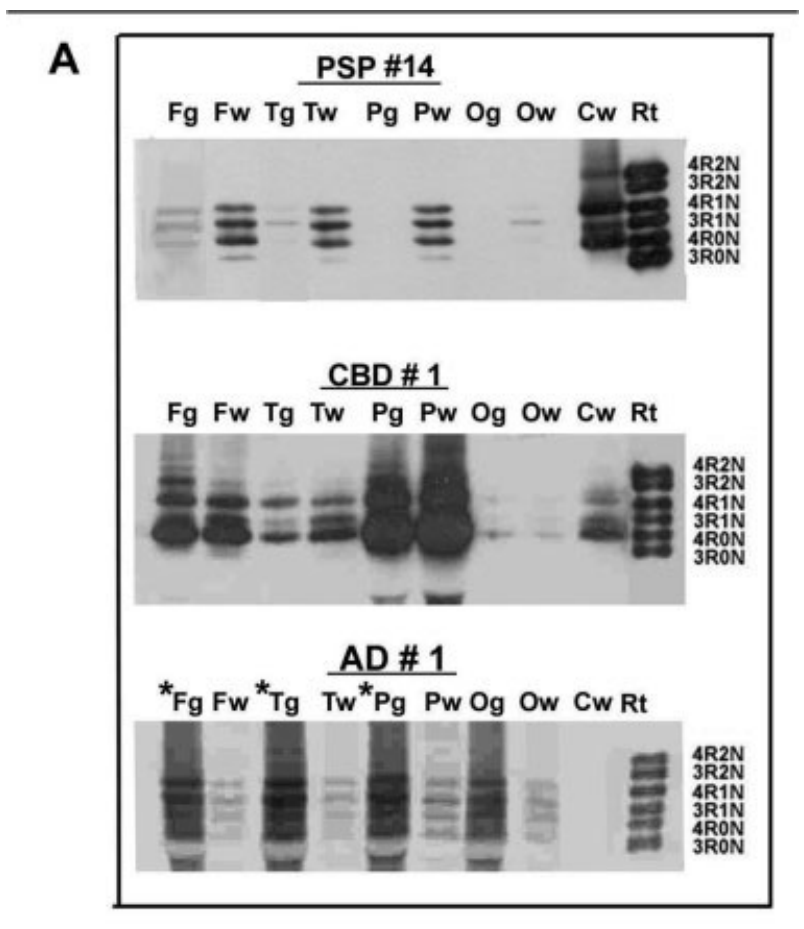

B

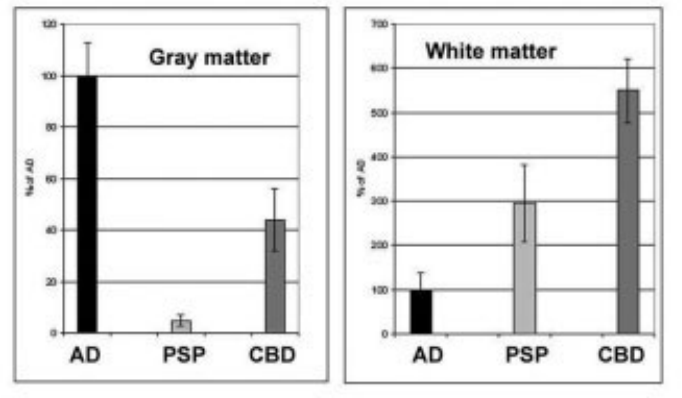

Fig 4. Comparative analysis of sarkosyl-insoluble tau in progressive supranuclear palsy (PSP), corticobasal degeneration $(C B D)$, and Alzheimer's disease (AD). (A) Representative Western blots of sarkosyl-insoluble tau proteins in $A D, C B D$, and PSP. To avoid the saturation of enzyme chemiluminescence (ECL) signal due to substantial differences in the amounts of pathological tau among cases, we loaded only 20\% of the material extracted from selected $A D$ samples compared with the PSP and CBD cases (asterisks). (B) Quantitative Western blot analysis was used to compare relative amounts of insoluble tau proteins in different neuropathological conditions. $F g=$ frontal cortex, gray matter; $F w=$ frontal cortex white matter; $O g=$ occipital cortex, gray matter; $O w=$ occipital cortex, white matter; $\mathrm{Pg}=$ parietal cortex, gray matter; $P w=$ parietal cortex, white matter; $T g=$ temporal cortex, gray matter; $T w=$ temporal cortex, white matter.

cal white matter was substantially higher than in the adjacent gray matter, PHF1 and AT8 immunoreactivity was significantly lower in the same samples, suggesting a reduction in the phosphorylation of selected phosphoepitopes in the white matter in PSP.

Finally, there were no differences in the apolipoprotein E genotypes or MAPT haplotypes among the PSP cases here with respect to the tau pathologies described earlier.

\section{Discussion}

In this study, we combined quantitative Western blot and IHC analyses of cortical and subcortical brain regions from 23 well-characterized PSP patients to characterize the profile and distribution pattern of tau pathologies and their isoform composition with particular emphasis on the gray versus white matter burden of these tau pathologies. Despite numerous comparative studies of PSP neuropathology, quantitative biochemical assessment of the distribution of tau pathology in gray versus white matter of multiple PSP brain regions has not been reported to complement information on microscopic tau lesions demonstrated by IHC. ${ }^{2,8,10,13,22}$ However, there is evidence that the biochemical detection of pathologically insoluble tau may not be reflected by microscopically evident tau lesions. ${ }^{12,13}$ Thus, our study was designed to address this issue in PSP by pursuing the multicenter collaborative investigation described here. Remarkably, although many of the observations in this large cohort of PSP cases confirmed previous studies of smaller numbers of PSP brains, ${ }^{2,8,10,13}$ we present novel data showing an unexpected abundance of biochemically detectable tau pathology in both white and gray matter despite that the insoluble tau in white matter was not apparent microscopically in sections of contiguous brain samples. Moreover, although we observed heterogeneity in the representation of pathological $3 \mathrm{R}$ and $4 \mathrm{R}$ tau isoforms in cortical versus subcortical regions across these PSP cases, biochemically detectable white matter tau pathology was a constant feature of PSP.

Although the burden of microscopic neocortical tau pathology correlates well with progressive cognitive decline and dementia in $\mathrm{AD}$, only mild or moderate amounts of cortical gray matter tau lesions may be seen microscopically in the brains of PSP patients with dementia. ${ }^{1,2,4,5,8,11}$ Although the burden of neurofibrillary tangles and glial tau pathology in PSP patients in this study with cognitive impairment was higher than in patients with relatively preserved memory and cognition, cortical tau lesions were sparse compared with abundant tau lesions in subcortical brain regions. The presence of previously undetected abundant white matter tau pathology demonstrated here by Western blots, but not by IHC, are significant for understanding the pathogenesis and clinical manifestations of PSP because they imply that the heretofore unappreciated white matter tau pathology we demonstrate biochemically in PSP could underlie cognitive and motor impairments in PSP. These findings also may have diagnostic implications for PSP if, for example, they can be exploited in neuroimaging 
studies to detect white matter abnormalities that facilitate distinction of PSP from other clinically similar neurodegenerative disorders. However, further studies are needed to test these hypotheses.

Biochemically, the amounts of sarkosyl-insoluble tau in the white matter were higher than in adjacent gray matter in the majority of PSP cases, and this also has been described in $\mathrm{CBD}^{9}$ and Pick's disease. ${ }^{12}$ However, although the biochemical findings in CBD correlate well with the abundance of tau-immunopositive lesions detected by IHC in gray and white matter of CBD, ${ }^{9}$ and similar correlations apply to PSP gray matter, enigmatically, this is not the case for white matter tau pathology in PSP.

Initially, the biochemical profile of pathological tau isoforms in PSP cortex was thought to be composed almost exclusively of $4 \mathrm{R}$ tau isoforms similar to $\mathrm{CBD}$, but our data here, and other recent reports, indicate
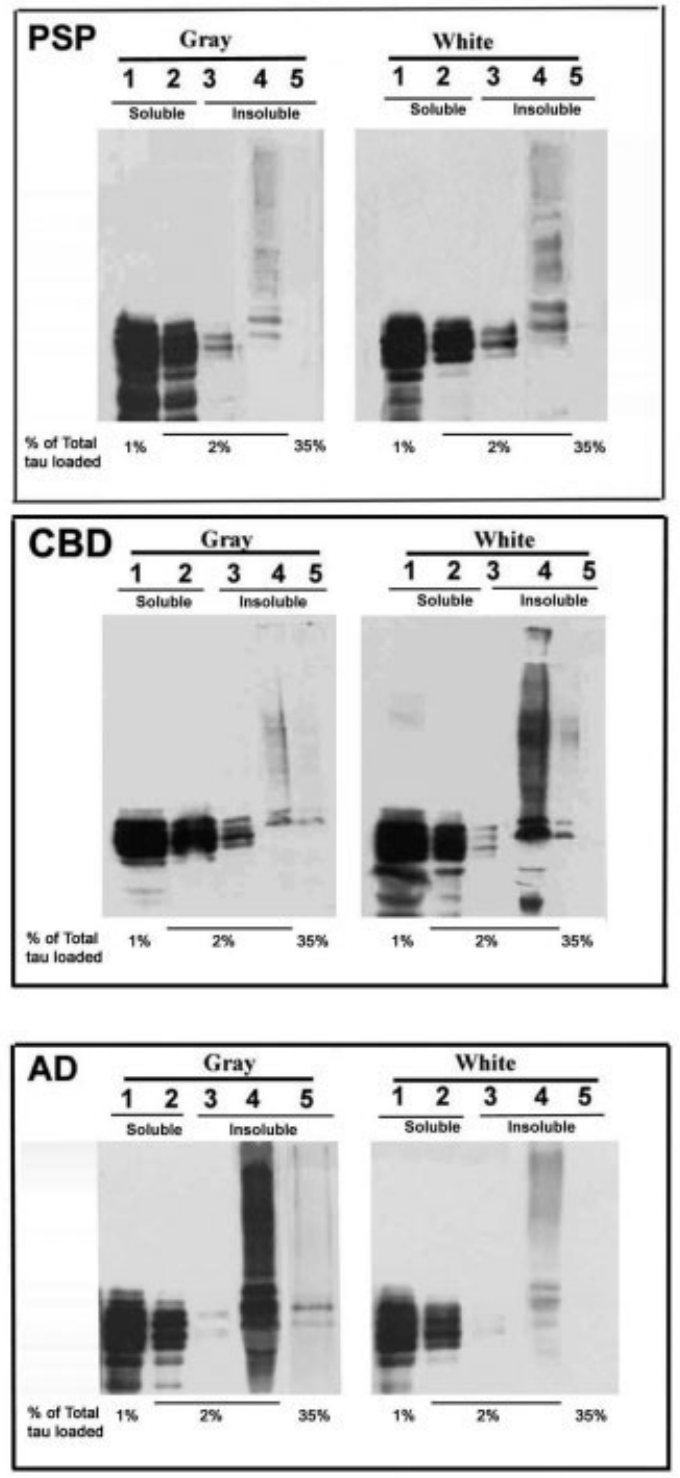

that there may be a variable mixture of pathological $3 \mathrm{R}$ - and 4R- tau isoforms in some PSP and CBD brains. $^{2,8,9}$ Based on our results, all PSP cases examined here could be divided into two major groups as a function of their cortical profile of pathological tau isoforms: (1) those with variable mixture of $3 \mathrm{R}$ and $4 \mathrm{R}$ tau isoforms, and (2) those with predominantly $4 \mathrm{R}$ tau isoforms. In contrast, $4 \mathrm{R}$ tau isoforms were significantly overrepresented in subcortical regions and brainstem regardless of the tau isoform profile in cortex of the same PSP brains.

Although there is a well-documented association between sporadic PSP and single nucleotide polymorphisms in the MAPT gene, similar associations have been reported for sporadic CBD, and it is unclear how these single nucleotide polymorphisms influence the onset or progression of PSP or the biochemical profile of pathological tau in PSP. ${ }^{2,21,22}$ Consistent with these earlier observations, 21 of our 23 cases were found to have an $\mathrm{H} 1 / \mathrm{H} 1$ and $\mathrm{A} 0 / \mathrm{A} 0$ MAPT single nucleotide polymorphisms profile.

In conclusion, PSP is characterized by a distinct biochemical profile of pathological tau proteins, and al-

Fig 5. Tau solubility profile in progressive supranuclear palsy (PSP), corticobasal degeneration (CBD), and Alzheimer's disease (AD). Here, $0.3 \mathrm{gm}$ of frozen temporal cortex from PSP and $A D$ brains were used for sequential extraction of tau with buffers/solutions of increasing stringency in the following sequence: lane $1=$ Buffer $A(0.75 \mathrm{M} \mathrm{NaCl}, 50 \mathrm{mM}$ tris(hydroxymethyl)aminomethane buffer, $p H$ 7.4, $2 m M$ EDTA); lane $2=1 \%$ Triton in Buffer A; lane 3 = radioimmunoprecipitation assay buffer; lane $4=2 \%$ sodium dodecyl sulfate (SDS); lane $5=70 \%$ formic acid (FA). Each extraction step was repeated 2 times with same buffer/solution, and after each extraction, samples were spun at 45,000g for 30 minutes at $4{ }^{\circ} \mathrm{C}$ so that aliquots from the resulting supernatants could be subjected to Western blot analysis using a mixture of monoclonal antibodies (MAbs) Tau14 and Tau46. Due to the significant differences in tau immunoreactivity between the extracts, the amount of sample used for analysis was adjusted as a percentage of the total extractable tau (soluble + insoluble) to avoid saturating the Western blot signal for some lanes so that lane 1 is $1 \%$ of total, lanes 2 to 4 are 2\% of total, and lane 5 is 35\% of total. Equal amounts from each extract were loaded for PSP, CBD, and AD. Samples from the first extraction step are for PSP, CBD, and AD. (Top) The tau solubility profile in PSP shows gray and white matter separately. Note that the amount of $2 \%$ SDS soluble tau proteins is higher in the white matter than in the corresponding gray matter. No detectable immunoreactivity was found in the FA extract. (Middle) Sequential extraction from a CBD case also is shown. (Bottom) Soluble and insoluble tau pool in $A D$ is shown. Although the amounts of 2\% SDS and FA-soluble tau are substantially higher in the gray matter compared with PSP and $C B D$, only a fraction of this signal is detected in the underlying white matter in $A D$. 


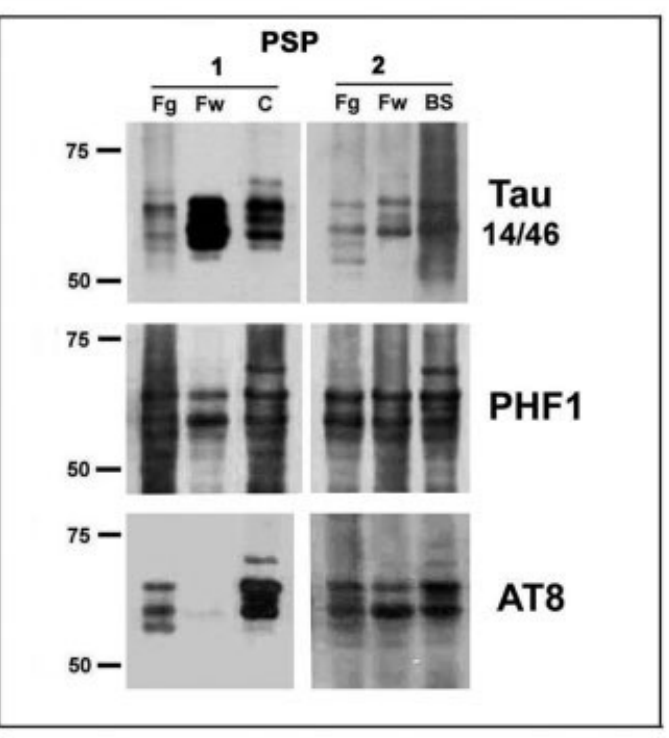

Fig 6. Tau phosphoepitope analysis in progressive supranuclear palsy (PSP). Nondephosphorylated samples from a sarkosylinsoluble fraction purified from the frontal cortex, gray and white matter separately, and cerebellum were subjected to Western blot analysis, and nitrocellulose membranes were probed with a mixture of phosphorylation-independent $\left(P_{i}\right)$ monoclonal antibodies (MAbs) T14 and T46 to demonstrate the total amount of insoluble tau proteins in each sample. Each membrane was stripped and reprobed with $P_{d}$ MAbs PHF1 and AT8. Note the distinct tau phosphorylation patterns for Tau14146, PHF1, and AT8 in white versus gray matter of cortex; AT8 phosphoepitopes representation in the same samples was more variable among patients. Molecular weight markers are shown on the left. $B S=$ brainstem; $C=$ cerebellum (dentate nucleus); $\mathrm{Fg}=$ frontal cortex, gray matter; $F w=$ frontal cortex, white matter.

though this is similar to $\mathrm{CBD}$, white matter tau pathology is far more abundant in CBD compared with PSP. Although white matter tau pathology is seen in other tauopathies in addition to PSP and CBD, it remains unclear what role glial and white matter tau pathology plays in mechanisms of brain degeneration in these tauopathies. However, it is likely that further insight into these issues could lead to better strategies for the diagnosis and treatment of PSP and related neurodegenerative tauopathies.

This work was supported by the NIH (National Institute of Aging, AG-10124, J.G.T.; AG08671, S.G.; AG-17586, V.M.-Y.L.), the Oxford Foundation, and the Marian S. Ware Alzheimer Program of the University of Pennsylvania. V.M-Y.L. is the John H. Ware 3rd Professor for Alzheimer's Disease Research, and J.Q.T. is the William Maul Measy-Truman G. Schnabel Jr. M.D. Professor of Geriatric Medicine and Gerontology.

We thank the families of patients who made this research possible, Dr M. Forman for helpful comments and discussions, and B.J. Cannon for technical assistance.

\section{References}

1. Cairns NJ, Lee VM-Y, Trojanowski JQ. Frontotemporal dementias: genetics and neuropathology. In: Miller B, Cummings JL, eds. The human frontal lobes. 2nd ed. (in press).

2. Dickson D. Sporadic tauopathies: Pick's disease, corticobasal degeneration, progressive supranuclear palsy and argyrophilic grain disease. In: Esiri M, Lee VM-Y, Trojanowski JQ, eds. The neuropathology of dementia. 2nd ed. Cambridge, UK: Cambridge University Press, 2004:227-256.

3. Forman MS, Lee VM-Y, Trojanowski JQ. Nosology of Parkinson's disease: looking for the way out of a quackmire. Neuron 2005;47:479-482.

4. McKhann GM, Albert MS, Grossman M, et al. Clinical and pathological diagnosis of frontotemporal dementia: Report of Work Group on Frontotemporal Dementia and Pick's Disease. Arch Neurol 2001;58:1803-1809.

5. Buee L, Bussiere T, Buee-Scherrer V, et al. Tau protein isoforms, phosphorylation and role in neurodegenerative diseases. Brain Res Rev 2000;33:1-36.

6. Lee VM-Y, Goedert M, Trojanowski JQ. Neurodegenerative tauopathies. Annu Rev Neurosci 2001;24:1121-1159.

7. Roy S, Zhang B, Lee VM-Y, Trojanowski JQ. Axonal transport defects: a common theme in neurodegenerative diseases. Acta Neuropathol 2005;109:5-13.

8. Buee L, Delacourte A. Comparative biochemistry of tau in progressive supranuclear palsy, corticobasal degeneration, FTDP-17 and Pick's disease. Brain Pathol 1999;9:681-693.

9. Forman MS, Zhukareva V, Bergeron C, et al. Signature tau neuropathology in gray and white matter of corticobasal degeneration. Am J Pathol 2002;160:2045-2053.

10. Takahashi M, Weidenheim KM, Dickson DW, Ksiezak-Reding H. Morphological and biochemical correlations of abnormal tau filaments in progressive supranuclear palsy. J Neuropathol Exp Neurol 2002;61:33-45.

11. Tan CF, Piao YS, Kakita A, et al. Frontotemporal dementia with co-occurrence of astrocytic plaques and tufted astrocytes, and severe degeneration of the cerebral white matter: a variant of corticobasal degeneration? Acta Neuropathol 2005;109: $329-338$.

12. Zhukareva V, Mann D, Pickering-Brown S, et al. Sporadic Pick's disease: a tauopathy characterized by a spectrum of pathological tau isoforms in gray and white matter. Ann Neurol 2002;51:730-739.

13. Zhukareva V, Trojanowski JQ, Lee VM-Y. Assessment of pathological tau proteins in frontotemporal dementias: qualitative and quantitative approaches. Am J Geriatr Psychiatry 2004; 12:136-145.

14. Forman MS, Farmer J, Johnson JK, et al. Frontotemporal dementia: clinicopathological correlations. Ann Neurol 2006; 59:952-962.

15. Grossman M, Farmer J, Leight $S$, et al. Cerebrospinal fluid profile in frontotemporal dementia and Alzheimer's disease. Ann Neurol 2005;57:721-729.

16. Mott RT, Dickson DW, Trojanowski JQ, et al. Neuropathologic, biochemical and molecular characterization of the frontotemporal dementias. J Neuropathol Exp Neurol 2005;64: $420-428$.

17. Roberson ED, Hesse JH, Rose KD, et al. Frontotemporal dementia progresses to death faster than Alzheimer disease. Neurology 2005;65:719-725. 
18. Zhukareva V, Shah K, Uryu K, et al. Biochemical analysis of tau proteins in argyrophilic grain disease, Alzheimer's disease and Pick's disease: a comparative study. Am J Pathol 2002;161:1135-1141.

19. Higuchi $M$, Ishihara $T$, Zhang $B$, et al. Transgenic mouse model of tauopathies with glial pathology and nervous system degeneration. Neuron 2002;35:433-446.

20. Addya K, Wang YL, Leonard DG. Optimization of apolipoprotein E genotyping. Mol Diagn 1997;2:271-276.
21. Baker M, Litvan I, Houlden H, et al. Association of an extended haplotype in the tau gene with progressive supranuclear palsy. Hum Mol Genet 1999;8:711-715.

22. Liu WK, Le TV, Adamson J, et al. Relationship of the extended tau haplotype to tau biochemistry and neuropathology in progressive supranuclear palsy. Ann Neurol 2001;50: 494-502. 